\title{
Experiencing a Mirrored World with Geotagged Social Media in Geollery
}

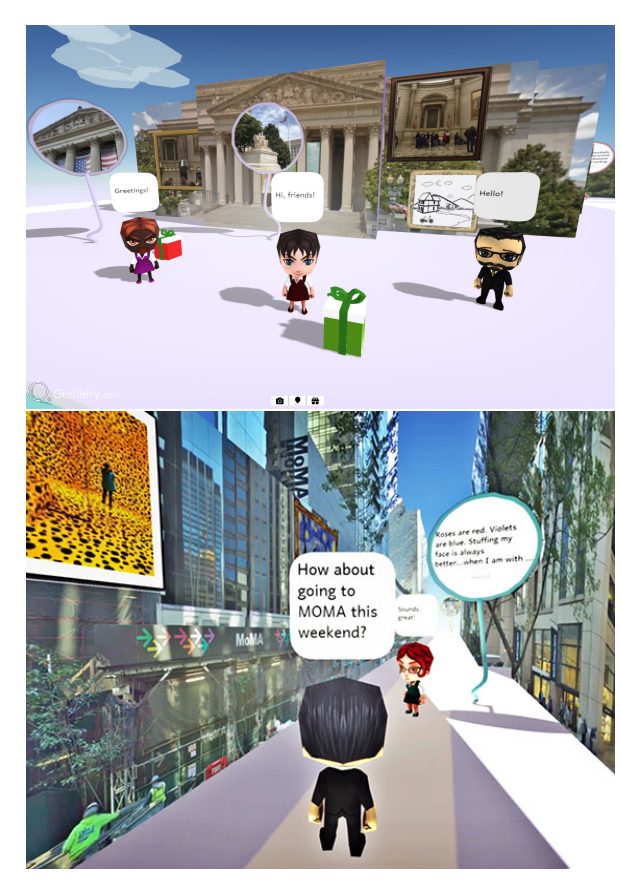

Figure 1: Geollery creates an interactive mirrored world in real time, in which users are immersed with 3D buildings, live chats, and geotagged social media. The social media are visualized as balloons, billboards, framed photos, and gift boxes, all in real time.

\author{
Ruofei Du, David Li, Amitabh Varshney \\ Augmentarium, UMIACS, and Computer Science Department \\ University of Maryland, College Park, MD, USA \\ Contact:me@duruofei.com,varshney@cs.umd.edu
}

\section{ABSTRACT}

We demonstrate the online deployment of Geollery [5], a mixed reality social media platform. We introduce an interactive pipeline to reconstruct a mirrored world at two levels of detail: the street level and the bird's-eye view. Instead of using offline 3D reconstruction approaches, our system streams and renders a mirrored world in real time, while depicting geotagged social media as billboards, balloons, framed photos, and virtual gifts. Geollery allows multiple users to see, chat, and collaboratively sketch with the spatial context in this mirrored world. We demonstrate a wide range of use cases including crowdsourced tourism, interactive audio guides with immersive spatial context, and meeting remote friends in mixed reality. We envision Geollery will be inspiring and useful as a standalone social media platform for those looking to explore new areas or looking to share their experiences. Please refer to https://geollery.com for the paper and live demos.

\section{ACM Reference Format:}

Ruofei Du, David Li, Amitabh Varshney. 2019. Experiencing a Mirrored World with Geotagged Social Media in Geollery. In CHI Conference on Human Factors in Computing Systems Extended Abstracts (CHI'19 Extended Abstracts), May 4-9, 2019, Glasgow, Scotland UK. ACM, New York, NY, USA, 4 pages. https://doi.org/10.1145/3290607.3313273

Permission to make digital or hard copies of part or all of this work for personal or classroom use is granted without fee provided that copies are not made or distributed for profit or commercial advantage and that copies bear this notice and the full citation on the first page. Copyrights for third-party components of this work must be honored. For all other uses, contact the owner/author(s)

CHI'19 Extended Abstracts, May 4-9, 2019, Glasgow, Scotland UK

() 2019 Copyright held by the owner/author(s).

ACM ISBN 978-1-4503-5971-9/19/05.

https://doi.org/10.1145/3290607.3313273 


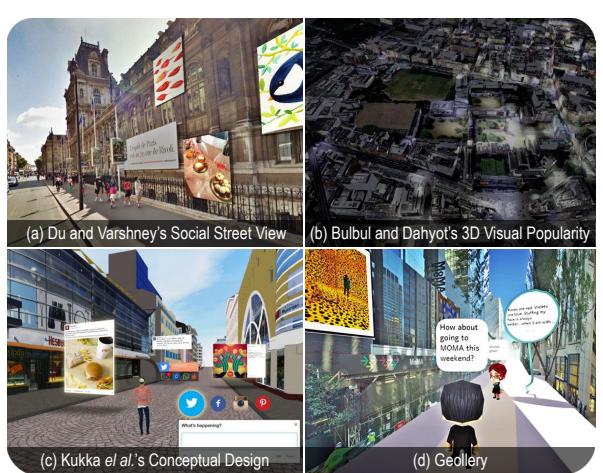

Figure 2: Comparison amongst mixed reality systems or designs for visualizing geotagged social media. (a) shows Social Street View [7], a real-time system which depicts social media as billboards via maximal Poisson-disk sampling [10], (b) shows Bulbul and Dahyot's offline system [1] which leverages virtual lighting to visualize popularity and sentiments of social media, (c) shows the conceptual design by Kukka et al. [11], which explores presentation manner, visibility, organization, and privacy during co-design activities, and (d) shows our results in Geollery, which fuses 3D textured buildings, geotagged social media, and virtual avatars in real time.

${ }^{1} \mathrm{~A}$ mirrored world is defined as "a representation of the real world in digital form [which] attempts to map real-world structures in a geographically accurate way" [12].

\section{INTRODUCTION}

Despite the rapid innovation in virtual and augmented reality recently (VR and AR), existing social media platforms typically use a linear narrative (Twitter, Facebook) or a grid layout (Pinterest). While these traditional layouts are efficient for quickly browsing through social media posts, they lack the spatial context associated with social media posts in immersive environments.

Recently, Social Street View [7,8] and its successors [1, 11] (Figure 2) have emerged for visualizing social media in mirrored worlds ${ }^{1}[9]$. Nevertheless, user interaction is limited to street-level panoramas thereby limiting the system to areas covered by street views. In addition, designing an interactive social platform with immersive geographical environments remains a challenge due to the real-time constraints of rendering 3D buildings. In addition, the design space of visualizing and interacting with social media in mixed reality settings is not yet fully explored.

In this demo paper, we present Geollery, a social mixed reality platform for creating, sharing, and exploring geotagged social media in a highly scalable, cloud-based infrastructure (Amazon Web Services). In addition to the design choices introduced in [5], we introduce the latest implementation of Geollery and demonstrate new visualization inspired by renovated algorithms.
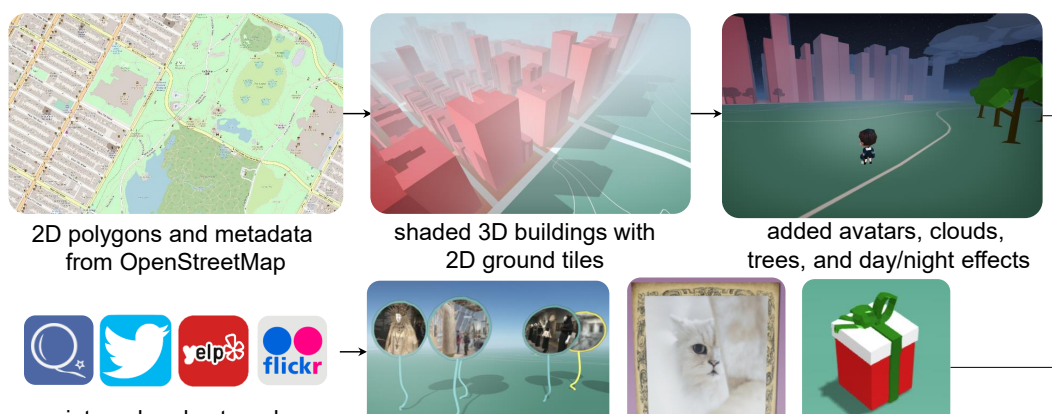

internal and external geotagged social media
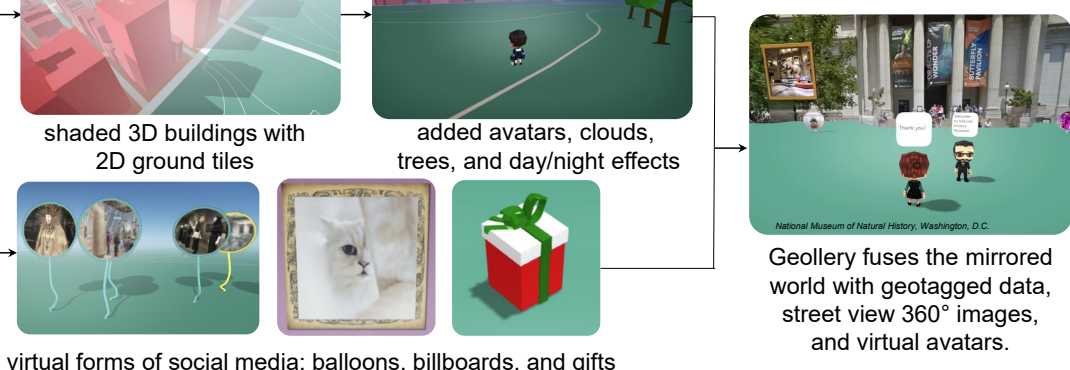

Geollery fuses the mirrored world with geotagged data street view $360^{\circ}$ images and virtual avatars.

Figure 3: The workflow of Geollery. Based on users' geospatial requests, Geollery loads the nearby 2D map tiles, extrudes 3D geometries, and renders social media in real time. We take advantage of WebGL to enable users to access Geollery via modern browsers on a desktop, a mobile phone, or a head-mounted display. 

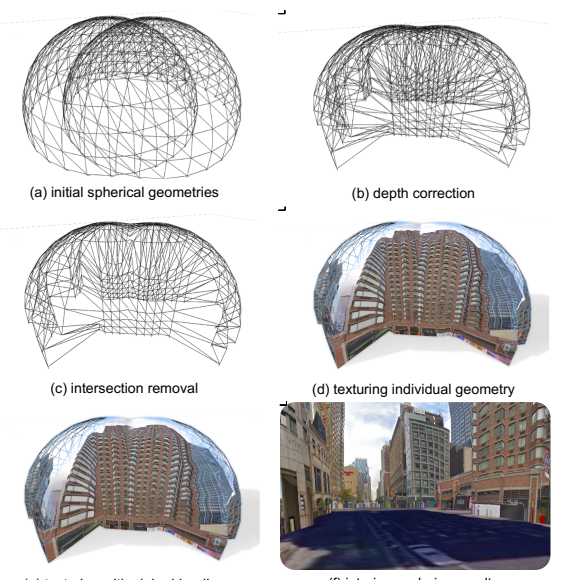

(f) interior rendering results

Figure 4: Our rendering pipeline for fusing $360^{\circ}$ images in fine detail. (a) We start by generating spherical geometries to represent the $360^{\circ}$ images. The segments of the sphere are greatly reduced for visualizing the geometries clearly. (b) In the vertex shader, we correct the depth value for each vertex by sampling the depth maps with its spherical coordinates. (c) In the fragment shader, we discard the pixels in the intersection of the adjacent geometries. (d) Texturing individual spheres with the corresponding 360 images may leave a visible seam. (e) Texturing the spheres with a weighted average according to the position to the camera yields a smoother result. (f) Finally, we interactively texture the ground plane with the corresponding satellite images, apply Gaussian filters in occluded regions, and allow the users to freely walk along the street while streaming the next street views on the go.

${ }^{2}$ Three.js: http://www.threejs.org.

\section{IMPLEMENTATION}

In its current form, Geollery is a web-based application, which is available on desktop, mobile phones, and virtual reality headsets. The architecture of Geollery consists of a 3D world generator, a social media scraper, a distributed SQL database, a web-server powered by Apache and PHP, and optional modules such as a temporal filter, a geospatial filter, and a computer-vision-based face filter.

We present the workflow of Geollery in Figure 3. Geollery consists of a data engine which streams 2D polygons and labels from OpenStreetMap, and social media data from our internal database and external sources such as Twitter, Yelp, and Flickr. Our rendering system is powered by Three.js ${ }^{2}$ with custom shaders. Geollery further allows users to explore social media nearby or at a custom location.

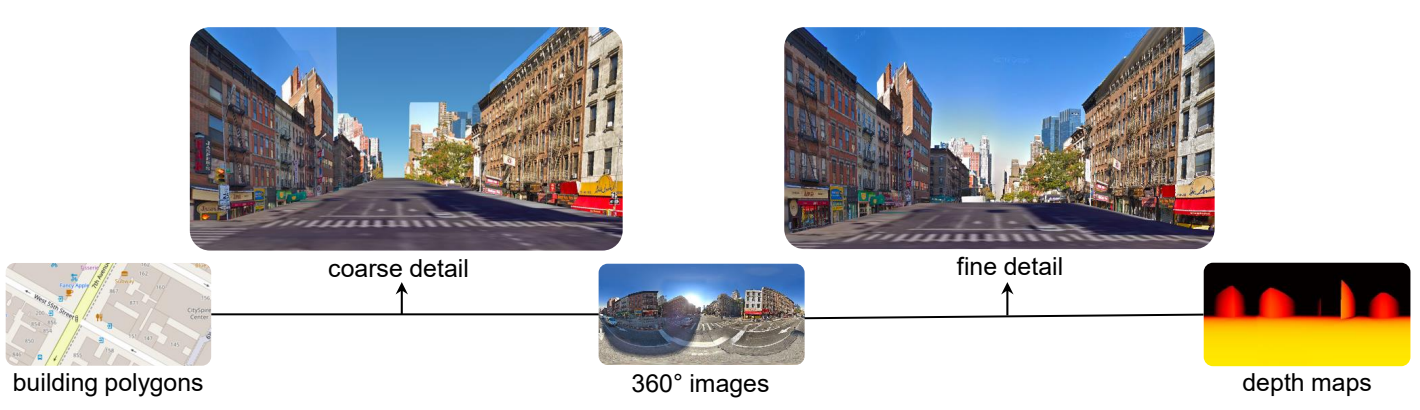

Figure 5: Results and overview of our interactive reconstruction pipeline at two levels of detail.

Unlike the prior art which aims to reconstruct the entire city, Geollery leverages a progressive approach to partially build the mirrored world at two levels of detail, as introduced in Figure 5. Given a pair of latitude and longitude coordinates, our pipeline streams and caches depth maps, street view panoramas, and building polygons from Google Maps and OpenStreetMap APIs. At a fine level of detail for close-up views, we render textured meshes using adjacent local street views and depth maps. The details are illustrated in Figure 4 and [6]. When viewed from afar, we apply projection mappings to 3D geometries extruded from building polygons for a coarse level of detail. Users could scroll in desktop or pinch to zoom on mobile devices. In contrast to teleportation, our system allows users to virtually walk through the mirrored world at the street level on various platforms.

In dense urban areas, undesirable cars and pedestrians often appear in street view images and get projected to the ground. To eliminate distorted cars and pedestrians, we choose to overlay Google Maps' satellite images instead. As shown in Figure 6, texturing the ground plane with satellite images results in a better visual appearance for our system. 


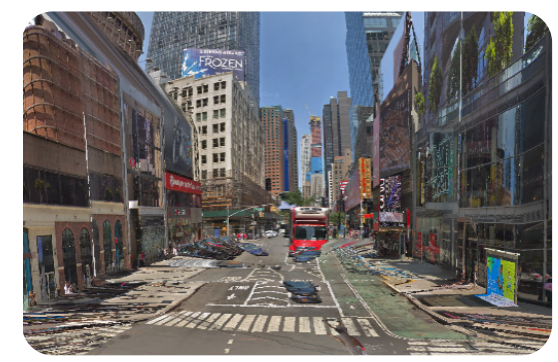

(a) texturing with street view images

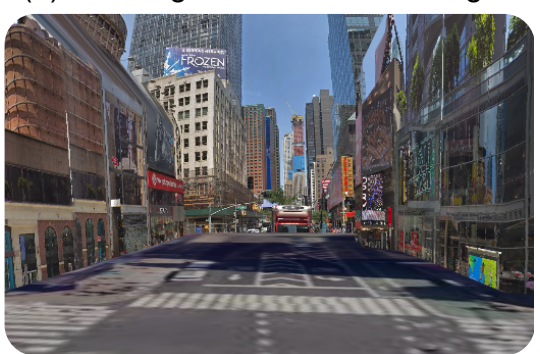

(b) texturing adding satellite images

Figure 6: Results before and after texturing with satellite images for the ground plane. Note that the satellite textures eliminate the distorted vehicles and pedestrians projected onto the road.

\section{CONCLUSION AND FUTURE VISION}

We have presented Geollery, a mixed reality social media platform. Geollery offers an opportunity to overcome one of the biggest challenges of social media today: lack of social interactions in a natural real-world setting that we are all familiar and comfortable with. Imagine: part social meetups, part social storytelling, part social city guide, part social gift exchange, all in a 3D world setting. Geollery is a web-based application that helps you discover nearby social media, keep up with your friends while using social psychology and gamification techniques to encourage and reward users for experiencing social interactions in a 3D setting. To users, Geollery is a tool for interacting with friends in a geographical context and rewarding oneself for discovering restaurants and navigating cities. To brands and advertisers, such a system may encourage and reward customers for participating in experiences that take place in the real world. In the future, we envision a real-time system that fuses street views, social media, and multiview videos $[3,4]$ to create a vivid mirrored world [2].

\section{REFERENCES}

[1] Abdullah Bulbul and Rozenn Dahyot. 2017. Social Media Based 3D Visual Popularity. Computers \& Graphics 63 (2017), 28-36. https://doi.org/10.1016/j.cag.2017.01.005

[2] Ruofei Du. 2018. Fusing Multimedia Data Into Dynamic Virtual Environments. Ph.D. Dissertation. University of Maryland, College Park.

[3] Ruofei Du, Ming Chuang, Wayne Chang, Hugues Hoppe, and Amitabh Varshney. 2018. Montage4D: Interactive Seamless Fusion of Multiview Video Textures . In Proceedings of ACM SIGGRAPH Symposium on Interactive 3D Graphics and Games (I3D). ACM, Montreal, Quebec, Canada, 124-133. https://doi.org/10.1145/3190834.3190843

[4] Ruofei Du, Ming Chuang, Wayne Chang, Hugues Hoppe, and Amitabh Varshney. 2019. Montage4D: Real-Time Seamless Fusion and Stylization of Multiview Video Textures. Journal of Computer Graphics Techniques 1, 15 (17 Jan. 2019), 1-34.

[5] Ruofei Du, David Li, and Amitabh Varshney. 2019. Geollery: a Mixed Reality Social Media Platform. In Proceedings of the 2019 CHI Conference on Human Factors in Computing Systems (CHI). ACM, 13. https://doi.org/10.1145/3290605.3300915

[6] Ruofei Du, David Li, and Amitabh Varshney. 2019. Interactive Fusion of $360^{\circ}$ Images for a Mirrored World. In 2019 IEEE Conference on Virtual Reality and 3D User Interfaces.

[7] Ruofei Du and Amitabh Varshney. 2016. Social Street View: Blending Immersive Street Views With Geo-Tagged Social Media. In Proceedings of the 21st International Conference on Web3D Technology (Web3D). ACM, 77-85. https://doi.org/10 $1145 / 2945292.2945294$

[8] Ruofei Du and Amitabh Varshney. 2016. Systems, Devices, and Methods for Generating a Social Street View. US Patent App. 15/559,955., 15 pages.

[9] David Gelernter. 1993. Mirror Worlds: Or: The Day Software Puts the Universe in a Shoebox... How It Will Happen and What It Will Mean. Oxford University Press.

[10] Cheuk Yiu Ip, M. Adil Yalçin, David Luebke, and Amitabh Varshney. 2013. PixelPie: Maximal Poisson-Disk Sampling With Rasterization. In Proceedings of the 5th High-Performance Graphics Conference (HPG '13). ACM, 17-26.

[11] Hannu Kukka, Minna Pakanen, Mahmoud Badri, and Timo Ojala. 2017. Immersive Street-Level Social Media in the 3D Virtual City: Anticipated User Experience and Conceptual Development. In Proceedings of the 2017 ACM Conference on Computer Supported Cooperative Work and Social Computing. ACM, 2422-2435.

[12] Wade Roush. 2007. Second Earth. Technology Review (2007), 38. 